\title{
Detection of Activated Parietal Epithelial Cells on the Glomerular Tuft Distinguishes Early Focal Segmental Glomerulosclerosis from Minimal Change Disease
}

\author{
Bart Smeets, ${ }^{*}$ Fabien Stucker, ${ }^{\dagger \dagger}$ Jack Wetzels, ${ }^{\S}$ Isabelle Brocheriou, ${ }^{\dagger}$ Pierre Ronco, ${ }^{\dagger}$ Hermann-Josef Gröne, ${ }^{\llbracket}$ Vivette D’Agati, \\ Agnes B. Fogo, ${ }^{* *}$ Toin H. van Kuppevelt, ${ }^{\dagger \dagger}$ Hans-Peter Fischer, ${ }^{\ddagger \ddagger}$ Peter Boor, ${ }^{\S \S}$ Jürgen Floege, ${ }^{*}$ Tammo Ostendorf, ${ }^{*}$ and \\ Marcus J. Moeller*
}

\begin{abstract}
From the Division of Nephrology and Clinical Immunology, * Department of Internal Medicine II, and the Q1 platform of the TRR57, ${ }^{\S 5}$ Institute for Pathology, RWTH Aachen University Hospital, Aachen, Germany; Department of Pathology, ${ }^{\dagger}$ INSERM U702, Hôpital Tenon, Paris, France; the Nephrology Service, ${ }^{\ddagger}$ University Hospitals of Geneva (HUG), Geneva, Switzerland; the Department of Nephrology, ${ }^{\S}$ and the Department of Biochemistry, ${ }^{\dagger \dagger}$ NCMLS, Radboud University Nijmegen Medical Centre, Nijmegen, The Netherlands; the Department of Cellular and Molecular Pathology, "German Cancer Research Center, Heidelberg, Germany; the Department of Pathology, "Columbia University, College of Physicians and Surgeons, New York, New York; the Department of Pathology, Microbiology and Immunology, ** Vanderbilt University Medical Center, Nashville, Tennessee; and the Q1 platform of the TRR57, Pathology, Sigmund-Freud-Straße 25, Bonn, Germany
\end{abstract}

\author{
Accepted for publication \\ August 22, 2014. \\ Address correspondence to \\ Marcus J. Moeller, Ph.D., \\ M.D., Department of \\ Nephrology and Clinical \\ Immunology, RWTH Univer- \\ sity Hospital Aachen, Pau- \\ welsstr. 3, 52074 Aachen, \\ Germany. E-mail: mmoeller@ \\ ukaachen.de.
}

\begin{abstract}
In rodents, parietal epithelial cells (PECS) migrating onto the glomerular tuft participate in the formation of focal segmental glomerulosclerosis (FSGS) lesions. We investigated whether immunohistologic detection of PEC markers in the initial biopsies of human patients with first manifestation of idiopathic nephrotic syndrome with no immune complexes can improve the sensitivity to detect sclerotic lesions compared with standard methods. Ninety-five renal biopsies were stained for claudin-1 (PEC marker), CD44 (activated PECS), and LKIV69 (PEC matrix); 38 had been diagnosed as early primary FSGS and 57 as minimal change disease. PEC markers were detected on the tuft in $87 \%$ of the biopsies of patients diagnosed as primary FSGS. PEC markers were detected in FSGS lesions from the earliest stages of disease. In minimal change disease, no PEC activation was observed by immunohistology. However, in $25 \%$ of biopsies originally diagnosed as minimal change disease the presence of small lesions indicative of a sclerosing process were detected, which were undetectable on standard periodic acid-Schiff staining, even though only a single histologic section for each PEC marker was evaluated. Staining for LKIV69 detected lesions with the highest sensitivity. Two novel PEC markers A-kinase anchor protein 12 and annexin A3 exhibited similar sensitivity. In summary, detection of PECs on the glomerular tuft by immunostaining improves the differentiation between minimal change disease and primary FSGS and may serve to guide clinical decision making. (Am J Pathol 2014, 184: 3239-3248; http://dx.doi.org/10.1016/j.ajpath.2014.08.007)
\end{abstract}

Primary or idiopathic focal segmental glomerulosclerosis (FSGS) is a leading cause of nephrotic syndrome in both adults and children, with a high risk of progression to endstage renal disease.$^{1-3}$ FSGS is characterized by the presence of mesangial sclerosis, obliteration of glomerular capillaries with adhesions between the glomerular tuft and Bowman's capsule, and epithelial cell hypertrophy and hyperplasia. ${ }^{4}$ Minimal change disease (MCD) is also a major cause of nephrotic syndrome in children and adults, but in contrast to FSGS glomerular sclerosing lesions are absent. ${ }^{5,6}$ By light microscopy, no abnormalities of the glomeruli are observed, hence the name of the disease. However, by electron microscopy marked podocyte foot process effacement can be observed in both entities.

\footnotetext{
Supported by the eRARE consortium "Rare-G" grant 01 GM 1208A (M.J.M.), German Research Foundation (DFG) grant BO 3755/2-1 (B.S. and P.B.), the NephCure Foundation grant F001 (B.S. and M.J.M.), and the Genzyme Renal Innovation Program (GRIP) (B.S. and M.J.M.). H.-P.F., P.B., J.F., T.O., and M.J.M. are members of the SFB/Transregio 57 DFG consortium "Mechanisms of organ fibrosis" (TP17, TP25, and Q1).

Disclosures: None declared.
} 
Given the similar clinical presentation in MCD and FSGS, the diagnosis is usually made by the pathologist who evaluates whether sclerotic lesions are present in the glomerular tuft. The differentiation between these disease syndromes has profound implications for treatment and prognosis. In general, primary FSGS is associated with a high risk of progression to end-stage renal disease and is therefore treated with more intense immunosuppression. ${ }^{7}$ However, the distinction between MCD versus early FSGS may be difficult, particularly when biopsy samples contain only a few glomeruli or when glomerular injury is at an early stage. ${ }^{8}$

We have shown that parietal epithelial cells (PECs) are crucially involved in the development of glomerulosclerosis. ${ }^{9-11}$ On an initial glomerular injury, PECs on Bowman's capsule can become activated. ${ }^{10,12}$ Activated PECs have a distinct phenotype, such as increased migration, proliferation, and/or matrix deposition of PECs. In general, activated PECs have a larger cuboidal cytoplasm with enlarged nuclei and form a thickened parietal basement membrane secondary to matrix production. ${ }^{9}$ Activated PECs can be recognized by the de novo expression of CD44 as found in experimental models of FSGS and crescentic glomerulonephritis and in biopsies of human patients with FSGS and crescentic glomerulonephritis. ${ }^{10,12,13}$ In experimental rodents it has been unequivocally found that in the process of development glomerulosclerosis, activated PECs invade the glomerular tuft at the site of an adhesion and cover long stretches of the tuft surface. In this process activated PECs deposit matrix. In humans, it is likely that a similar sequence of events occurs in the development of glomerulosclerotic lesions, albeit definite experimental evidence (eg, lineage tracing) is not available, and conclusions are based largely on immunohistologic markers.

Here, we assessed whether detection of invading activated PECs on the glomerular tuft can be used as a novel diagnostic marker for early FSGS lesions and thus can distinguish MCD from early FSGS in human patients. To this end, a large panel of biopsies with early FSGS or MCD was collected from five pathologic centers in Europe and the United States. A single section of each biopsy was immunostained for one of three different PEC markers, that is, CD44, claudin-1, and LKIV69. CD44 is the main receptor for osteopontin and hyaluronic acid and is involved in cell adhesion, matrix interaction, and migration. ${ }^{14}$ Activated PECs show de novo expression of CD44 and can be present within FSGS lesions and on Bowman's capsule, often in close proximity of an adhesion. ${ }^{9,10}$ In a recent study we have shown that a de novo expression of CD44 is a rare finding in MCD. ${ }^{13}$ Claudin-1 is a tight junction molecule that we believe is expressed exclusively by PECs within the glomerulus. ${ }^{15}$ LKIV69 is a single-chain antibody that within the glomerulus recognizes particular heparan sulfate moieties present in matrix produced specifically by PECs. ${ }^{16}$ The latter two markers are constitutively expressed by PECs; therefore, they can be used to detect PEC invasion of the glomerular tuft. ${ }^{10}$ Normal glomeruli will show immunostaining of both markers exclusively at the Bowman's capsule. In contrast, in FSGS

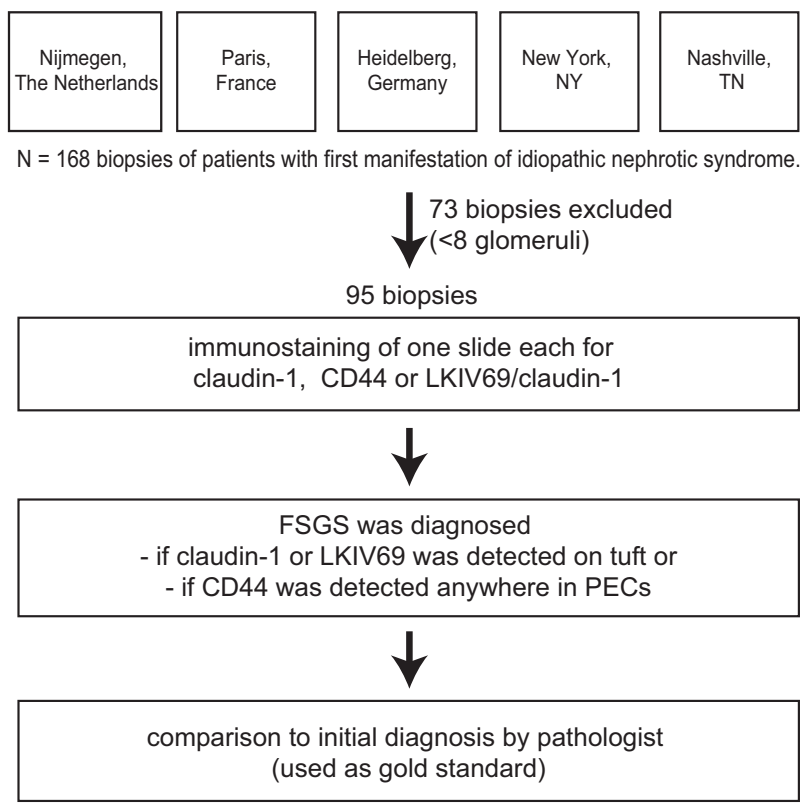

Figure 1 The flow chart describes the study design (sources of biopsies along with the analysis protocol [immunostaining, focal segmental glomerulosclerosis (FSGS) diagnosis, and comparison to initial diagnosis by pathologist])

lesions these markers will also stain the PECs or their matrix in a visceral location. We postulated that such aberrant staining could be an early indicator of FSGS lesions, preceding overt sclerosis detectable by light microscopy.

\section{Materials and Methods}

\section{Biopsies}

Kidney biopsies were received from the following five pathology centers: i) Departments of Pathology and Nephrology, Radboud University Medical Center, Nijmegen, The Netherlands; ii) INSERM U702, Hôpital Tenon, Paris, France; iii) Department of Cellular and Molecular Pathology, German Cancer Research Center, Heidelberg, Germany; iv) Department of Pathology, Columbia University, College of Physicians and Surgeons, New York, NY; and v) Department of Pathology, Microbiology, and Immunology, Vanderbilt University Medical Center, Nashville, TN. The analyzed kidney biopsies were from adult ( $42 \pm 20$ years) patients with a diagnosis of MCD or FSGS. All patients exhibited nephrotic range proteinuria at the time of biopsy. Immunofluorescence studies found no immune complex deposits. Electron microscopy was performed within the different institutes for the diagnosis of MCD and primary FSGS. Of note, the FSGS biopsies were selected not to have advanced glomerulosclerosis. The histologic analyses of the present study were approved by the local ethical committees.

\section{Immunohistochemistry}

Paraffin sections ( $4 \mu \mathrm{m})$ were deparaffinized, exposed to heat antigen retrieval in citrate-based antigen unmasking solution 

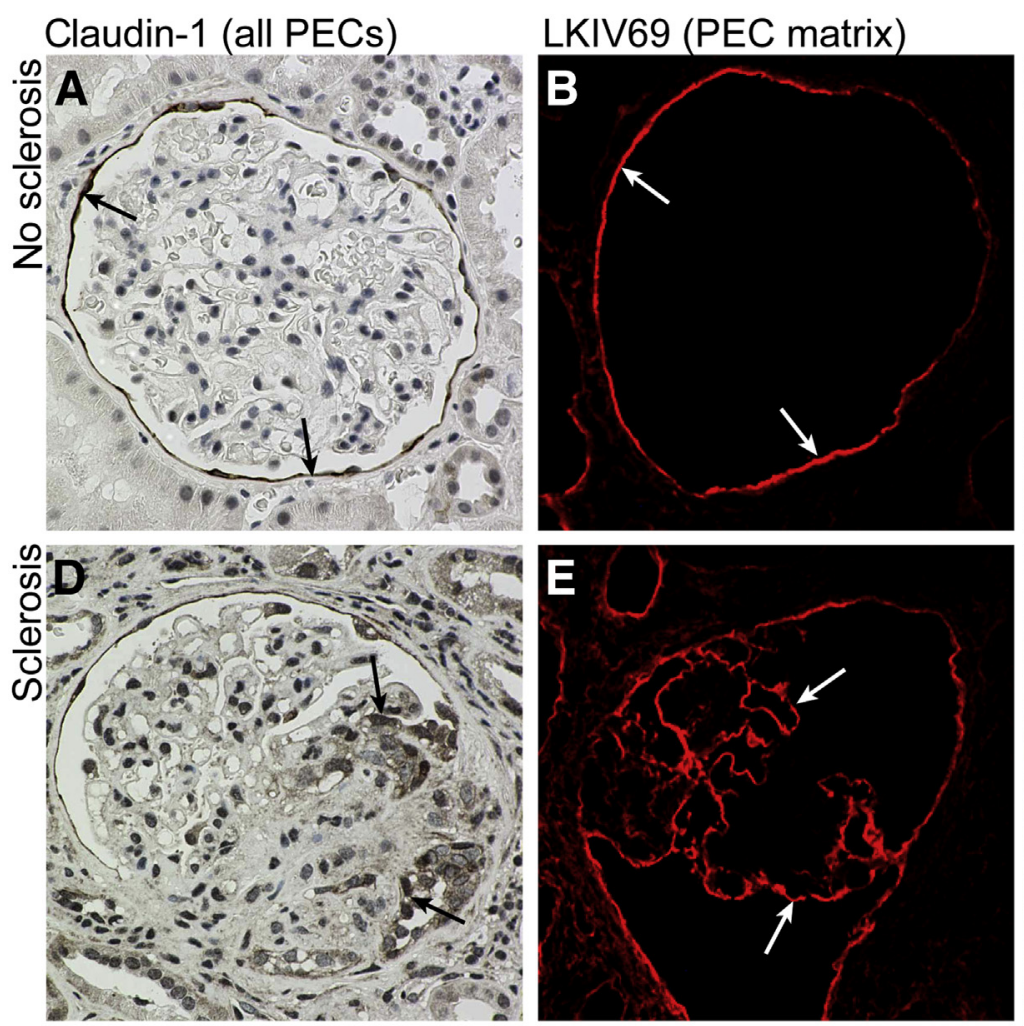

CD44 (activated PECs)
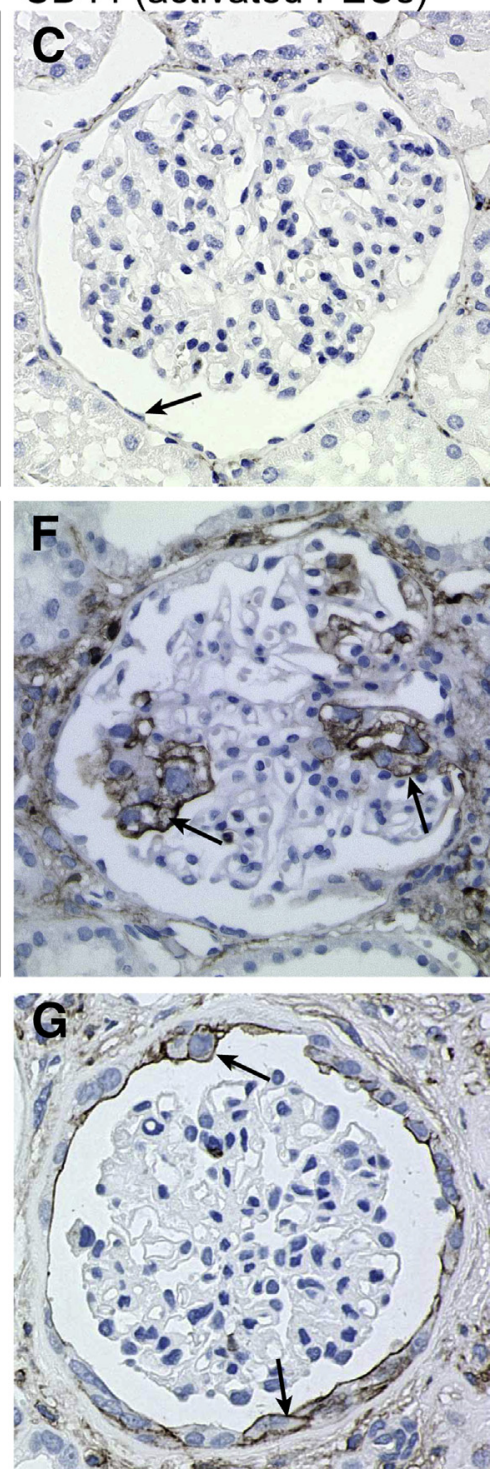

Figure 2 Expression of claudin-1, LKIV69, and CD44 in normal glomeruli and in glomerulosclerosis. A-C: Claudin-1, LKIV69, and CD44 immunostaining in normal glomeruli of a normal human kidney (macroscopically normal renal tissue obtained from a patient undergoing nephrectomy because of renal cell carcinoma). Claudin-1 and LKIV69 stain all parietal epithelial cells (PECs) (A, arrows) or their matrix of Bowman's capsule (B, arrows), respectively. In normal glomeruli, CD44 is not expressed (C, arrow). D-F: Invading PECs in focal segmental glomerulosclerosis (FSGS) co-express claudin-1 (D, arrows), LKIV69 (E, arrows), and CD44 (F, arrows). G: In some FSGS cases, glomeruli without a visible glomerulosclerotic lesion show activated PECs on Bowman's capsule. Activated PECs are enlarged and express CD44 de novo (G, arrows). A, C, D, F, and G: Nuclei were counterstained with Mayer's hematoxylin solution.

(Vector Laboratories Inc., Burlingame, CA), and incubated with anti-claudin-1 (Abcam, Cambridge, UK) or anti-CD44 (Abcam) primary antibody. The secondary antibody was a biotinylated goat anti-rabbit or a biotinylated goat anti-mouse antibody (Vector Laboratories Inc.), respectively. Detection was performed with vectastain $\mathrm{ABC}$ kit (Vector Laboratories Inc.) with the use of peroxidase as label and 3,3'-diaminobenzidine as substrate. Nuclei were counterstained with Mayer's hematoxylin solution (Sigma-Aldrich, St. Louis, MO). To examine the histology of the biopsies some of the paraffin sections were stained with periodic acid-Schiff (PAS).

\section{Immunofluorescence}

A double immunofluorescence staining for $\operatorname{LKIV} 9^{16}$ and claudin-1 (Abcam) was performed on $4-\mu \mathrm{m}$ paraffin sections. The claudin-1 immunofluorescence staining was used to confirm the PEC origin of the LKIV69 ${ }^{+}$matrix. The primary antibodies were detected with an anti-VSV-Cy3 (SigmaAldrich) and an anti-rabbit Alexa Fluor 488 secondary antibody (Life Technologies GmbH, Darmstadt, Germany), respectively.

After the primary analysis, selected biopsies were stained with PAS and/or double or triple stained with antibodies for LKIV69 and A-kinase anchor protein 12 (AKAP12; Sigma-Aldrich) or for annexin A3 (ANXA3; Sigma-Aldrich) and synapotopodin (Santa Cruz Biotechnology, Santa Cruz, CA), respectively.

\section{Image Analysis}

Images were acquired with a Keyence BZ-9000 Microscope (Keyence Deutschland GmbH, Neu-Isenburg, Germany).

Unaware of the original diagnosis, the total number of glomeruli and the number of glomeruli with positive staining 
A
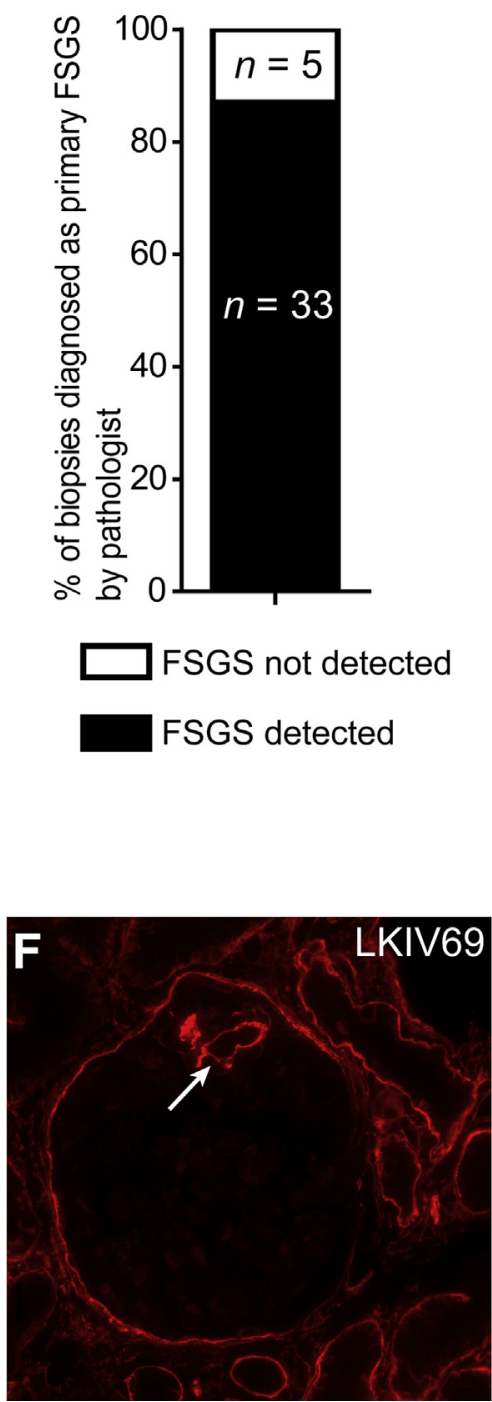
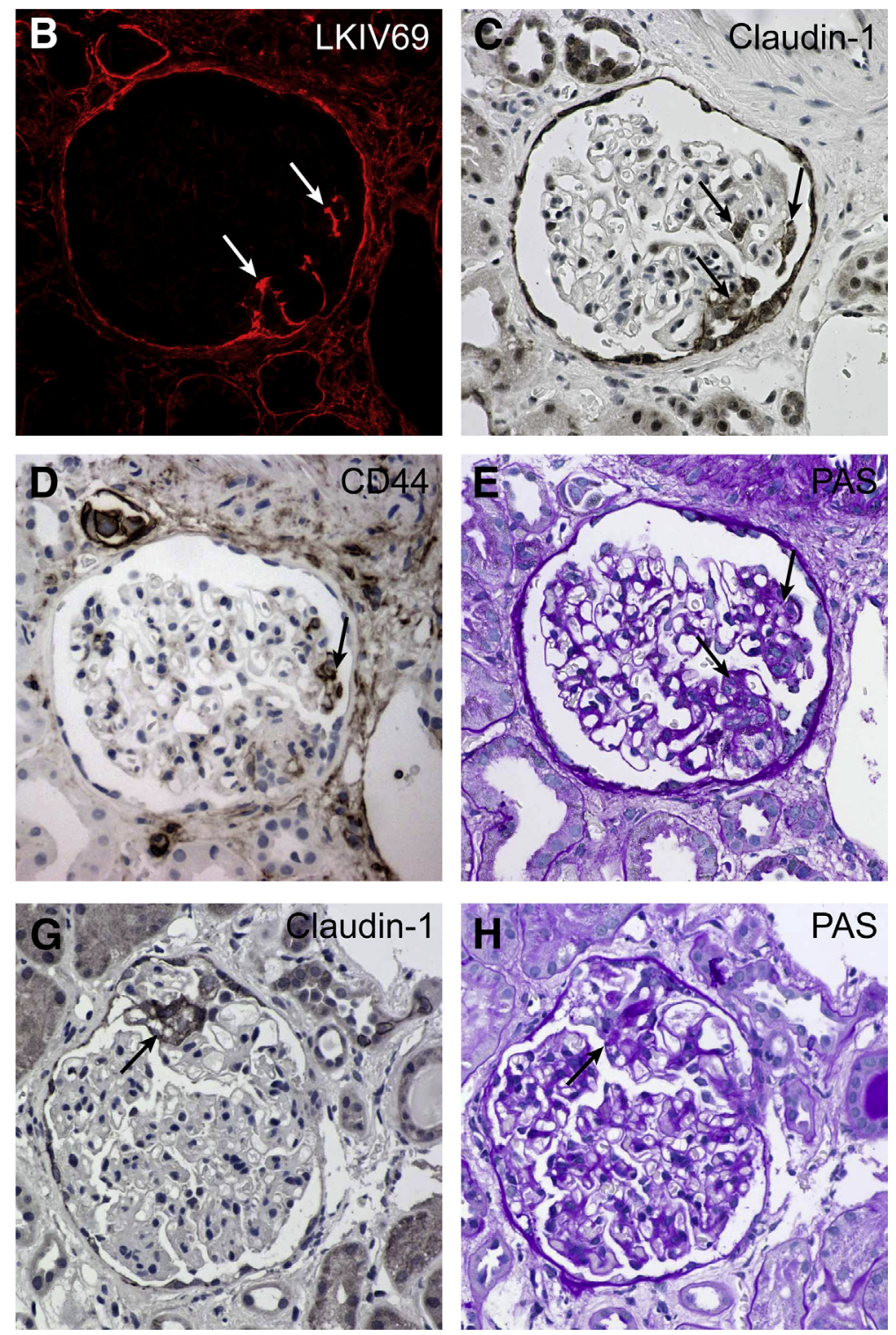

Figure 3 Focal segmental glomerulosclerosis (FSGS) can be diagnosed by the presence of PECs on the glomerular tuft. A: Evaluation of parietal epithelial cell (PEC) marker expression found visceral PECs in 33 of 38 biopsies (87\%) diagnosed with FSGS. B-H: Immunostaining of PEC markers (B, C, D, F, and G, arrows) found that PECs were involved in the development of FSGS lesions from the earliest stages of disease. Consecutive PAS-stained sections found small segmental lesions in the same location as the PEC markers (E and $\mathbf{H}$, arrows). C, D, and G: Nuclei were counterstained with Mayer's hematoxylin solution.

for any of the PEC markers were analyzed. Biopsies with one or both markers, claudin-1 or LKIV69, in a visceral location were assigned as presumptive FSGS. Any biopsy with CD44 expression by PECs (on Bowman's capsule and/or in visceral location) was assigned as FSGS. Those biopsies negative for the three PEC markers were classified as presumptive MCD. Diagnosis assignment by PEC immunostaining was compared with previous light microscopic diagnosis by a pathologist on different glomerular sections.

\section{Results}

A total of 168 biopsies of patients with no lesions in the original biopsy or patients with biopsy-proven early FSGS were immunostained for three markers expressed by PECs, that is, claudin-1, LKIV69, and CD44 (Figure 1). Only biopsies with eight or more glomeruli on a single section were included in this study $(n=95)$. In normal glomeruli without glomerulosclerosis, immunostaining for claudin-1 and LKIV69 stained exclusively along Bowman's capsule (Figure 2, A and B). ${ }^{10,16}$ Within FSGS lesions, both markers were also expressed by the invading PECs and can therefore be found in a visceral location ${ }^{10}$ (Figure 2, D and E). CD44 is normally not expressed by kidney epithelial cells (Figure 2C). Within affected glomeruli and in FSGS lesions, de novo expression of CD44 by activated PECs was observed (Figure 2, F and G). ${ }^{10,12,13,17}$ 
A

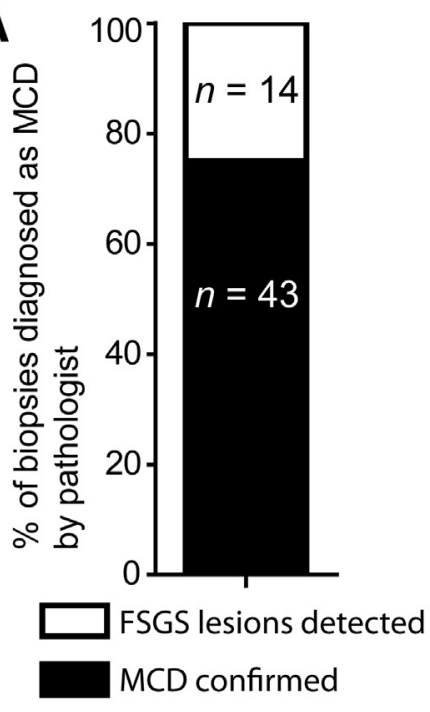

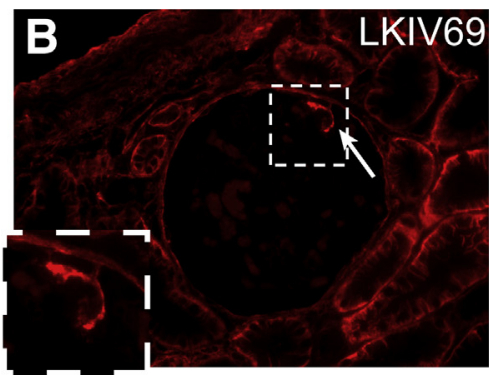
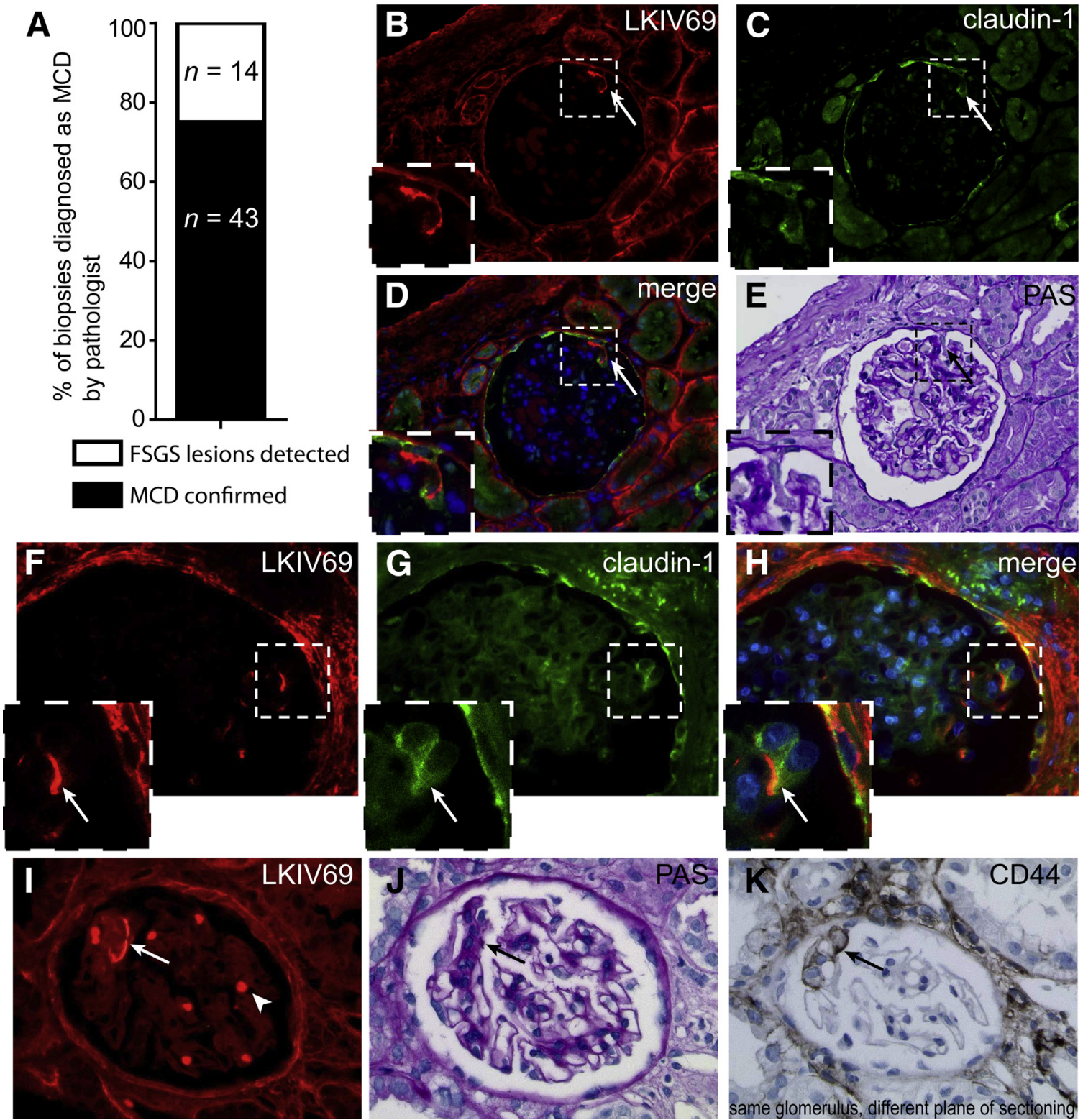

Figure 4 Focal segmental glomerulosclerosis (FSGS) lesions detected in biopsies originally diagnosed as minimal change disease (MCD). A: Evaluation of parietal epithelial cell (PEC) marker expression in biopsies diagnosed as MCD found no abnormal PECs in 43 of 57 biopsies (75\%). In 14 of 57 biopsies (25\%) biopsies, the presence of FSGS lesions was detected with the three PEC markers. B-K: FSGS lesions detected by immunostaining for PEC markers were typically small or nondetectable in PAS-stained serial sections (E and $\mathbf{J}$, arrows). PEC matrix marker LKIV69 was most sensitive (B, F, and $\mathbf{I}$, arrows). LKIV69 ${ }^{+}$matrix accumulations were often covered by claudin-1 and/or CD44 ${ }^{+}$PECS (C, D, G, H, and $\mathbf{K}$, arrows). B-H: The insets show a higher magnification of the small glomerular sclerotic lesion. In some sections autofluorescence of erythrocytes is visible (I, arrowhead). K: Nuclei were counterstained with Mayer's hematoxylin solution.

FSGS Can Be Diagnosed by the Presence of PECs on the Glomerular Tuft

To assess whether we can detect and diagnose FSGS exclusively by immunostaining for three PEC markers, the analysis of the PEC marker immunostaining was compared with the original diagnosis by the pathologist (Figure 1). Thirty-eight of the 95 biopsies were diagnosed by the pathologist with early FSGS. Analysis of PEC markers confirmed the presence of glomerulosclerosis in 33 of 38 biopsies (87\%) diagnosed with FSGS (Figure 3A). Five biopsies originally diagnosed as FSGS did not have positive immunostaining for LKIV69, claudin-1, or CD44.

Many FSGS lesions had positive immunostaining for all three PEC markers (Figure 3, B-E). After analysis of the PEC marker expression within the biopsies, PAS-stained consecutive sections were prepared (depending on availability of sufficient material). PAS-stained sections of the biopsies originally diagnosed with FSGS revealed the presence of glomerulosclerosis (Figure 3E) in the same location where visceral expression of PEC markers was detected (Figure 3, B-E). However, sclerotic lesions were 


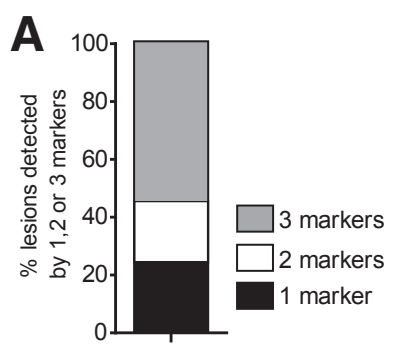

B

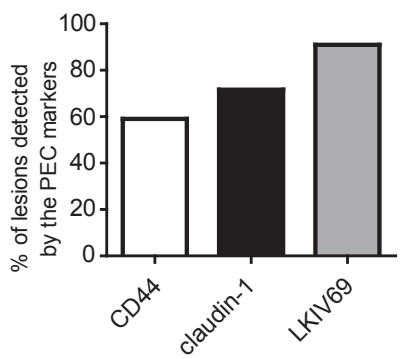

Figure 5 Analysis of the sensitivity of parietal epithelial cell (PEC) markers to detect sclerotic lesions. A: Graph shows that 55\% of the focal segmental glomerulosclerosis (FSGS) lesions within individual glomeruli were detected by all three PEC markers. The remaining $45 \%$ of the detected lesions were detected by either one $(24 \%)$ or two $(21 \%)$ markers. B: LKIV69 is the most sensitive marker because $90 \%$ of the detected lesions were LKIV69 ${ }^{+}$.

sometimes difficult to detect on PAS-stained sections without the knowledge of the immunostaining (Figure 3, $\mathrm{F}-\mathrm{H})$.

In general, LKIV69 was the most sensitive marker. Small sclerotic lesions in PAS staining were easily detected on LKIV69-stained sections in virtually all of the cases (Figure 3, F versus $\mathrm{H}$ ). In the representative glomerulus shown, claudin- $1^{+}$PECs can be detected in a visceral location (Figure 3G).

From our previous findings in murine experimental crescentic glomerulonephritis and FSGS ${ }^{10,12}$ it was anticipated to detect CD $44^{+}$PECs in FSGS lesions and along Bowman's capsule of affected glomeruli. However, in $94 \%$ of the CD $44^{+}$glomeruli, CD44 staining was restricted to the visceral PECs (Figure 3D). CD44 is expressed also by other cells, mainly macrophages and leukocytes. However, in most glomeruli the expression of CD44 by PECs could be confirmed by positive staining for LKIV69 and/or claudin-1 (Figure 3, B and C) by the same cells in consecutive sections. Of interest, LKIV69 and claudin-1 immunostaining in Figure 3, F and G, respectively, clearly indicated visceral PECs and matrix deposition. The PAS-stained section showed within the same segment enlarged epithelial cells and hyalinosis but no overt sclerosis (Figure $3 \mathrm{H}$ ). This observation indicates that PECs can be detected even in the early phase of sclerotic lesions.

\section{FSGS in Biopsies Originally Diagnosed as Minimal Change Disease}

When analyzing PEC marker expression on biopsies diagnosed as MCD, 43 of 57 biopsies (75\%) indicated no glomerular PEC marker expression, consistent with the original diagnosis. Interestingly, in 14 of 57 biopsies (25\%), immunostaining for one or more of the PEC markers was detected (Figure 4A). In most of these cases, immunostaining indicated small accumulations of PEC matrix (LKIV69) (Figure 4, B, F, and I), often colocalizing with claudin-1 (Figure 4, C, D, G, and $\mathrm{H}$ ) and/or $\mathrm{CD}_{4} 4^{+}$cells (Figure $4 \mathrm{~K}$ ) on the glomerular tuft. Of note, these small sclerotic lesions were not detectable in consecutive PAS-stained sections, prepared after the initial immunohistologic analysis (Figure 4, E and J). Thus, we postulate that cells expressing PEC markers and PEC matrix on the glomerular tuft could be an earlier marker than overt sclerosis; whether these patients follow a course indicative of FSGS will require further study.

\section{Immunostaining of PEC Matrix Is a Sensitive Tool for} Detection of Small FSGS Lesions

Analysis of the lesions detected by the PEC markers found that within most detected lesions all three markers were present $(55 \%)$ and that the remaining $45 \%$ of the detected lesions were detected by either one $(24 \%)$ or two $(21 \%)$ markers (Figure 5A). Comparison of the three markers found that LKIV69 was most sensitive and could be detected in $91 \%$ of the lesions detected by the marker staining (Figure 5B). Even small depositions of PEC matrix were easily detected by LKIV69 immunostaining. A summary of the immunohistologic PEC marker analysis compared with the original diagnosis by the pathologist is shown in Figure 6.

\section{Detecting PECs in Sclerotic Lesions by Using AKAP12 or ANXA3 as Alternative Markers}

So far, claudin-1 is the best established marker for the detection of PECs. In addition in the present study, claudin- 1 was a specific marker for the detection of PECs within the glomerulus. Nonetheless, claudin-1 is a tight junction molecule expressed at intercellular junctions. In thin paraffin sections, claudin-1 staining was relatively weak and/or punctated, somewhat limiting the utility of this marker. Therefore, better markers are needed.

Recently, AKAP12 was described as being specifically expressed in murine PECs. ${ }^{18}$ In our hands, anti-AKAP12 immunofluorescence staining resulted in strong immunostaining of human PECs and some tubular cells (Figure 7). A much weaker staining was observed in tubulointerstitial cells and

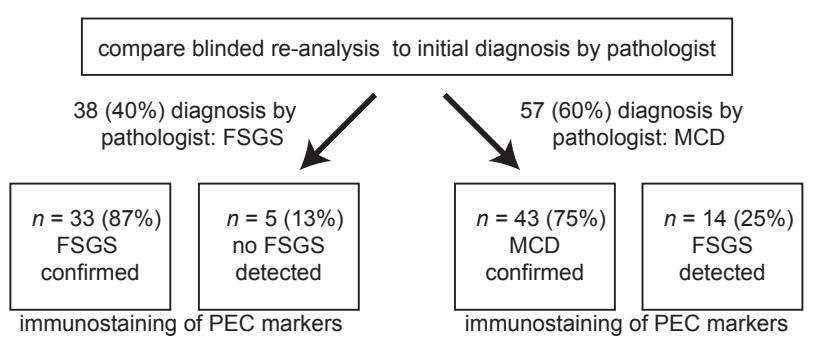

Figure 6 Summary of the results of the immunohistologic analysis of parietal epithelial cell (PEC) markers compared with the original diagnosis of the pathologist. FSGS, focal segmental glomerulosclerosis; MCD, minimal change disease. 

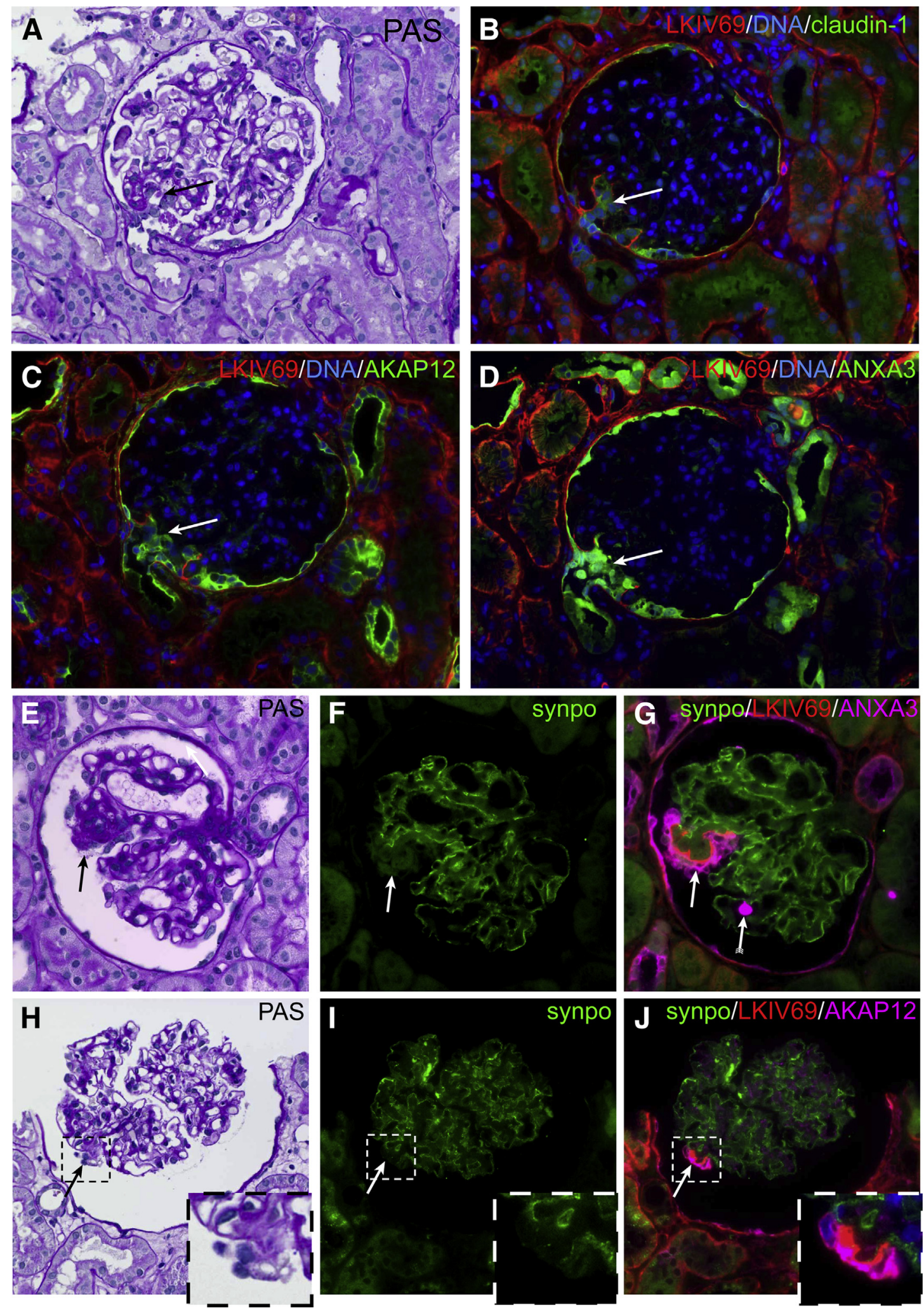

Figure 7 AKAP12 or annexin A3 (ANXA3) identifies parietal epithelial cells (PECs) in small focal segmental glomerulosclerosis (FSGS) lesions. A-D: Consecutive sections of a glomerulus show sclerosis at the glomerular tip (A, arrow). PAS staining (A); double-immunfluorescence staining of LKIV69 with claudin-1 (B), AKAP12 (C), or ANXA3 (D); and Hoechst as nuclear marker in blue. The FSGS lesion at the tip detected by LKIV69 (B-D) contains claudin- $1^{+}$, AKAP12 $2^{+}$, and ANXA3 ${ }^{+}$PECs (B-D, white arrows, respectively). The claudin-1 immunostaining is weaker than AKAP12 and ANXA3 immunostaining (B versus $\mathbf{C}$ and $\mathbf{D})$ ). E-J: Small lesions (E and H, arrows) can be detected by ANXA3 (F and G, arrows) and AKAP12 (I and J, arrows) immunostaining. Triple immunofluorescence for ANXA3/LKIV69/synaptopodin (synpo) and AKAP12/LKIV69/synpo shows that podocytes do not express ANXA3 or AKAP12. ANXA3 is also expressed by leukocytes (G, arrow with tails). H-J: The insets show a higher magnification. 
endothelial cells of glomerular and peritubular capillaries (Figure 7C). Second, in a previous study we detected ANXA3 in a specific population of proximal tubular cells of the kidney, the so-called scattered tubular cells. ${ }^{19,20}$ Scattered tubular cells express similar proteins as PECs. In the present study, we found that within the glomerulus ANXA3 was expressed exclusively by PECs and infiltrating leukocytes ${ }^{21}$ (Figure 7D). Both of these marker stainings were comparable at least to claudin-1 (Figure 7, B versus C and D). To assess whether these new PEC markers can also be used to detect small FSGS lesions, additional biopsies containing small FSGS lesions were analyzed. In all cases, small matrix adhesions on the glomerular tuft (as detected by LKIV69 staining) were covered by $\mathrm{AKAP}_{12}{ }^{+}$and $\mathrm{ANXA}^{+}$cells (Figure 7, E-J). These PECs cannot be identified as such in the PAS-stained consecutive section (Figure 7, A, E, and $\mathrm{H}$ ).

\section{Discussion}

Here, we assessed whether immunostaining for PEC markers can distinguish primary or idiopathic FSGS from MCD by increasing the sensitivity for the detection of small early lesions in the initial biopsy that may indicate development of FSGS.

Our study describes four major findings. First, detection of cells expressing PEC markers on the glomerular tuft indicates even small lesions and enabled us to confirm the diagnosis of FSGS in most biopsies. The high correlation between the PEC marker expression and the presence of glomerulosclerosis provides further strong evidence for the involvement of PECs in the final common pathway in every case of primary FSGS. ${ }^{9}$ In the remaining $13 \%$ (five biopsies), no PECs were detected on the glomerular tuft; they did not express activation marker CD44 either. Several explanations may explain why no FSGS lesions could be detected in these five biopsies. First is the focal and segmental nature of FSGS. The original diagnosis was made on other sections of the same biopsy than those analyzed in our laboratory. Although we examined only those biopsies with eight or more glomeruli, lesions could still be present in other glomeruli or in different planes of sectioning, especially because we focused on the detection of early FSGS cases in this study. Second, the original diagnosis is normally based on several serial sections of a single biopsy, thereby examining several planes of sectioning. We stained and analyzed only one biopsy section per PEC marker staining (ie, a total of three consecutive sections).

The second major finding was that in MCD most biopsies showed no PEC activation or PECs in a visceral location, confirming the original MCD diagnosis and confirming our recent findings. ${ }^{13}$ This further supports the notion that PEC activation and PECs invading the glomerular tuft are processes strictly associated with microanatomic abnormalities and can therefore be used to distinguish early FSGS from MCD.

The third major finding was that immunhistologic staining for PEC markers could detect sclerotic lesions in $25 \%$ of the biopsies originally diagnosed as MCD. The diagnosis MCD versus primary FSGS relies exclusively on the histologic findings in the biopsy, emphasizing the clinical relevance of the pathologic analysis. In general, the lesions detected by PEC markers were small and often located close to the glomerular tip (tip lesions). Of note, in the present study the primary pathologic diagnosis and the presented secondary PEC marker expression analysis have been performed on different glomerular planes/sections in early FSGS and MCD. The focal and segmental nature of FSGS, especially for these small lesions, may explain why the lesions were missed in the original sections evaluated by the pathologist. In fact, previous studies have noted that small lesions particularly at the glomerular tip may be missed in biopsies initially diagnosed as MCD. ${ }^{22,23}$ By analysis of the PEC markers, LKIV69 in particular, even small FSGS precursor lesions could be detected which would not have been noted on PAS-stained sections.

In the past decades, efforts have been made to improve pathologic discrimination between FSGS and MCD. These efforts are to improve the diagnosis and subsequent treatment of the diseases and to obtain a better understanding of the pathomechanisms of both diseases. It was proposed that differences in the podocyte expression of dystroglycans could discriminate between MCD and FSGS because only in MCD the expression of $\beta$-dystroglycan ${ }^{24}$ and $\alpha$-dystrogly$\mathrm{can}^{25}$ was reduced. This would also suggest that the pathomechanism underlying MCD may be different from that of FSGS. However, these studies have been debated because the findings in later studies by the same group ${ }^{26}$ and other groups $^{27,28}$ were not consistent with the previous studies. ${ }^{24,25}$ More recently it was found that urinary soluble CD80 (B7.1) is elevated in MCD and not in FSGS, which could be promising for both diagnosis and pathogenesis of the disease. $^{29}$

To discriminate MCD from FSGS, we focused on the role of PECs in the development and progression of FSGS. ${ }^{9-11}$ We hypothesized that even the earliest stages of FSGS could be distinguished from MCD by the presence of activated PECs on Bowman's capsule or of invading cells expressing PEC markers on the glomerular tuft. Recent findings in a study by Hodgin et $\mathrm{al}^{30}$ supported our model. In that study, the researchers performed molecular profiling on laser-captured glomerular tufts from patients with FSGS and collapsing glomerulopathy and compared the profiles to those of patients with MCD. ${ }^{30}$ Interestingly, approximately $46 \%$ (11 of 24) of the genes listed to be the highest up-regulated genes in the FSGS + collapsing glomerulopathy group are normally expressed by PECs. This is a striking finding because, in the latter study, lasermicrocaptured glomerular tufts were analyzed, and the number of PECs should be limited. This finding by Hodgin et $\mathrm{al}^{30}$ can be explained by migration of PECs onto the glomerular tuft and suggested that ectopic presence of PECs on the glomerular tuft can be used as a diagnostic marker for FSGS. 
In experimental models of FSGS, PEC activation on Bowman's capsule (ie, de novo expression of CD44) was a prominent feature. ${ }^{10}$ Therefore, we predicted that PEC activation on Bowman's capsule would be predictive for imminent development of a FSGS lesion. However, in the present study only $4 \%$ of the $\mathrm{CD} 44^{+}$glomeruli showed marker expression of CD44 on Bowman's capsule. Most of the $\mathrm{CD} 44^{+}$PECs were involved in FSGS lesions, suggesting a functional role of CD44 in the formation of the lesion, for example, PEC migration. Thus, FSGS could only be discriminated from MCD after PECs had formed cellular bridges/adhesions or had migrated onto the glomerular tuft. Even so, we observed that this was already seen in the earliest stages in which no overt sclerosis was visible on PAS-stained sections.

Finally, as the fourth major finding, the present study describes two novel markers expressed by PECs that allowed the detection of PECs with higher sensitivity in early FSGS lesions, that is, AKAP12 and ANXA3, compared with conventional claudin-1 staining. Today, the list of PEC markers is still limited, but especially in the past few years more PEC markers have been described. The detection of these new markers in healthy and or affected glomeruli may be valuable for diagnostic purposes and for the understanding of the molecular processes in PEC (patho)physiology.

Taken together, immunohistologic detection of PEC markers can be used to improve the sensitivity of the FSGS diagnosis. In particular, the detection of parietal matrix by using the LKIV69 antibody may be a sensitive tool to detect early FSGS lesions, which might otherwise be missed on PASstained sections.

\section{Acknowledgments}

B.S. performed staining and blinded analysis, analyzed data, and wrote the manuscript; F.S., J.W., I.B., P.R., H.-J.G., V.D'A., and A.B.F. selected and contributed biopsies; T.H.v.K. contributed reagents; H.-P.F. and P.B. performed staining; and J.F., T.O., and M.J.M. designed the study, analyzed data, and wrote the manuscript.

\section{References}

1. Haas M, Meehan SM, Karrison TG, Spargo BH: Changing etiologies of unexplained adult nephrotic syndrome: a comparison of renal biopsy findings from 1976-1979 and 1995-1997. Am J Kidney Dis 1997, 30: 621-631

2. Haas M, Spargo BH, Coventry S: Increasing incidence of focalsegmental glomerulosclerosis among adult nephropathies: a 20-year renal biopsy study. Am J Kidney Dis 1995, 26:740-750

3. Abrantes MM, Cardoso LS, Lima EM, Penido Silva JM, Diniz JS, Bambirra EA, Oliveira EA: Predictive factors of chronic kidney disease in primary focal segmental glomerulosclerosis. Pediatr Nephrol 2006, 21:1003-1012

4. Jennette JC, Olson JL, Schwartz MM, Silva FG: Focal segmental glomerulosclerosis. Heptinstall's Pathology of the Kidney. ed 6. Baltimore, Lippincott Williams \& Wilkins, 2007, pp 159-175
5. Cameron JS: The nephrotic syndrome and its complications. Am J Kidney Dis 1987, 10:157-171

6. Jennette JC, Olson JL, Schwartz MM, Silva FG: The nephrotic syndrome and minimal change disease. Heptinstall's Pathology of the Kidney. ed 6. Baltimore, Lippincott Williams \& Wilkins, 2007, pp $138-142$

7. D'Agati VD, Alster JM, Jennette JC, Thomas DB, Pullman J, Savino DA, Cohen AH, Gipson DS, Gassman JJ, Radeva MK, MoxeyMims MM, Friedman AL, Kaskel FJ, Trachtman H, Alpers CE, Fogo AB, Greene TH, Nast CC: Association of histologic variants in FSGS clinical trial with presenting features and outcomes. Clin J Am Soc Nephrol 2013, 8:399-406

8. Fuiano G, Comi N, Magri P, Sepe V, Balletta MM, Esposito C, Uccello F, Dal Canton A, Conte G: Serial morphometric analysis of sclerotic lesions in primary "focal" segmental glomerulosclerosis. J Am Soc Nephrol 1996, 7:49-55

9. Smeets B, Moeller MJ: Parietal epithelial cells and podocytes in glomerular diseases. Semin Nephrol 2012, 32:357-367

10. Smeets B, Kuppe C, Sicking EM, Fuss A, Jirak P, van Kuppevelt TH, Endlich K, Wetzels JF, Grone HJ, Floege J, Moeller MJ: Parietal epithelial cells participate in the formation of sclerotic lesions in focal segmental glomerulosclerosis. J Am Soc Nephrol 2011, 22: $1262-1274$

11. Dijkman H, Smeets B, van der Laak J, Steenbergen E, Wetzels J: The parietal epithelial cell is crucially involved in human idiopathic focal segmental glomerulosclerosis. Kidney Int 2005, 68: $1562-1572$

12. Smeets B, Uhlig S, Fuss A, Mooren F, Wetzels JF, Floege J, Moeller MJ: Tracing the origin of glomerular extracapillary lesions from parietal epithelial cells. J Am Soc Nephrol 2009, 20: 2604-2615

13. Fatima H, Moeller MJ, Smeets B, Yang HC, D’Agati VD, Alpers CE, Fogo AB: Parietal epithelial cell activation marker in early recurrence of FSGS in the transplant. Clin J Am Soc Nephrol 2012, 7:1852-1858

14. Goodison S, Urquidi V, Tarin D: CD44 cell adhesion molecules. Mol Pathol 1999, 52:189-196

15. Kiuchi-Saishin Y, Gotoh S, Furuse M, Takasuga A, Tano Y, Tsukita S: Differential expression patterns of claudins, tight junction membrane proteins, in mouse nephron segments. J Am Soc Nephrol 2002, 13: 875-886

16. Wijnhoven TJ, Lensen JF, Rops AL, van der Vlag J, Kolset SO, Bangstad HJ, Pfeffer P, van den Hoven MJ, Berden JH, van den Heuvel LP, van Kuppevelt TH: Aberrant heparan sulfate profile in the human diabetic kidney offers new clues for therapeutic glycomimetics. Am J Kidney Dis 2006, 48:250-261

17. Florquin S, Nunziata R, Claessen N, van den Berg FM, Pals ST, Weening JJ: CD44 expression in IgA nephropathy. Am J Kidney Dis 2002, 39:407-414

18. Burnworth B, Pippin J, Karna P, Akakura S, Krofft R, Zhang G, Hudkins K, Alpers CE, Smith K, Shankland SJ, Gelman IH, Nelson PJ: SSeCKS sequesters cyclin D1 in glomerular parietal epithelial cells and influences proliferative injury in the glomerulus. Lab Invest 2012, 92:499-510

19. Smeets B, Boor P, Dijkman H, Sharma SV, Jirak P, Mooren F, Berger K, Bornemann J, Gelman IH, Floege J, van d J, Wetzels JF, Moeller MJ: Proximal tubular cells contain a phenotypically distinct, scattered cell population involved in tubular regeneration. J Pathol 2013, 229:645-659

20. Berger K, Bangen JM, Hammerich L, Liedtke C, Floege J, Smeets B, Moeller MJ: Origin of regenerating tubular cells after acute kidney injury. Proc Natl Acad Sci U S A 2014, 111: $1533-1538$

21. Alcorta DA, Barnes DA, Dooley MA, Sullivan P, Jonas B, Liu Y, Lionaki S, Reddy CB, Chin H, Dempsey AA, Jennette JC, Falk RJ: Leukocyte gene expression signatures in antineutrophil cytoplasmic autoantibody and lupus glomerulonephritis. Kidney Int 2007, 72: 853-864 
22. Howie AJ, Pankhurst T, Sarioglu S, Turhan N, Adu D: Evolution of nephrotic-associated focal segmental glomerulosclerosis and relation to the glomerular tip lesion. Kidney Int 2005, 67:987-1001

23. Howie AJ, Kizaki T: Glomerular tip lesion in the 1962-66 Medical Research Council trial of prednisone in the nephrotic syndrome. Nephrol Dial Transpl 1993, 8:1059-1063

24. Regele HM, Fillipovic E, Langer B, Poczewki H, Kraxberger I, Bittner RE, Kerjaschki D: Glomerular expression of dystroglycans is reduced in minimal change nephrosis but not in focal segmental glomerulosclerosis. J Am Soc Nephrol 2000, 11:403-412

25. Giannico G, Yang H, Neilson EG, Fogo AB: Dystroglycan in the diagnosis of FSGS. Clin J Am Soc Nephrol 2009, 4:1747-1753

26. Giannico G, Phillips S, Shyr Y, Alpers CE, D’Agati VD, Fogo AB: Dystroglycan patterns in FSGS variants. Mod Pathol 2011, 24(suppl 1):344A-345A
27. Vogtlander NP, Dijkman H, Bakker MA, Campbell KP, van der Vlag J, Berden JH: Localization of alpha-dystroglycan on the podocyte: from top to toe. J Histochem Cytochem 2005, 53:1345-1353

28. Vogtlander NP, van der Vlag J, Bakker MA, Dijkman HB, Wevers RA, Campbell KP, Wetzels JF, Berden JH: Expression of sialidase and dystroglycan in human glomerular diseases. Nephrol Dial Transpl 2010, 25:478-484

29. Garin EH, Mu W, Arthur JM, Rivard CJ, Araya CE, Shimada M, Johnson RJ: Urinary CD80 is elevated in minimal change disease but not in focal segmental glomerulosclerosis. Kidney Int 2010, 78: 296-302

30. Hodgin JB, Borczuk AC, Nasr SH, Markowitz GS, Nair V, Martini S, Eichinger F, Vining C, Berthier CC, Kretzler M, D’Agati VD: A molecular profile of focal segmental glomerulosclerosis from formalinfixed, paraffin-embedded tissue. Am J Pathol 2010, 177:1674-1686 